\begin{tabular}{|l|l|l|l|l|}
\hline Revista Clío América & ISSN: 1909-941X & Vol. 8 & No. 16 & Julio - Diciembre de 2014 \\
\hline
\end{tabular}

\title{
El numeral 8 de la norma y la mejora continua en las empresas de Ibagué
}

\section{Standard item 8 and continue improvement in enterprises from Ibague}

Resumen: El proyecto de investigación se realizó a partir de la aplicación de un cuestionario estructurado, diseñado con base en la Norma ISO 9004, a siete empresas que habían sido certificadas, siendo una muestra definida por conveniencia. El artículo presenta los resultados del análisis de la aplicación del numeral 8 de la norma "Medición, análisis y mejora", resaltándose dos grandes conclusiones: el alto grado de autoapreciación que las empresas tienen de su gestión del Sistema de calidad y la aplicación, desarrollo y demostración de las acciones que ellas adelantan se enfocan a mejorar la eficacia de sus sistemas de calidad y a fortalecer su cultura de calidad.

Palabras clave: Calidad; Mejora continua; Numeral 8 Norma ISO.

\section{JEL Classification: M10}

\begin{abstract}
The research project was developed by a structured questionnaire application, designed IS0 9004 based-on, to seven certified enterprises that formed a sample chosen by convenience method. This paper presents the results of application of Standard numeral 8. Measuring, analysis and improve behavior analysis, emphasizing two great conclusions: the self-appreciation high level enterprises have about theirs Quality system management, and the application, development and demonstration about the actions they do are focused to improve their quality systems efficacy and to strengthen their quality culture.
\end{abstract}

Keywords: Quality; Continue improving; Standard IS0 numeral 8.

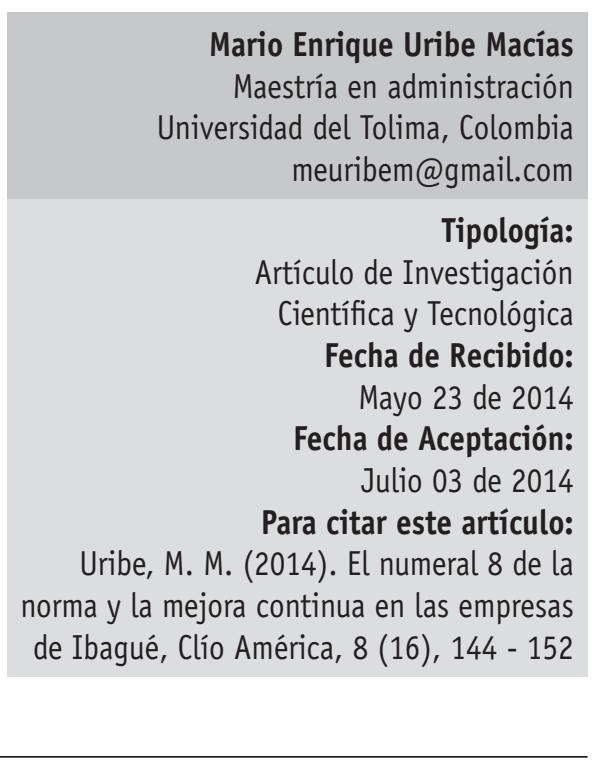

Clío América. Julio - Diciembre 2014, Vol. 8 No. 16, p.p. 144 - 152 


\section{Introducción}

Como parte de los resultados obtenidos en el proyecto de investigación "Caracterización de los sistemas de gestión de la calidad de empresas certificadas bajo la NTC-ISO 9001:2000 en la ciudad de Ibagué" (Uribe, 2010), el artículo presenta el análisis de la situación presentada en las empresas estudiadas con respecto al numeral 8 de la norma: Medición, análisis y mejora.

En primera instancia, el lector encontrará el propósito del estudio (en términos generales para el proyecto de investigación y específicamente para el interés de este artículo). A continuación se presenta la metodología utilizada en el proyecto de investigación y su derivación para el tópico específico de este artículo. Posteriormente se describen e interpretan los resultados del trabajo de campo, mediante el uso de 7 preguntas, con base en los lineamientos de la norma IS0 9004:2000 (que presenta las directrices para la mejora del desempeño del sistema de gestión de la calidad), directamente relacionadas con el ítem 8 de la norma; dentro del mismo capítulo se sintetizan los resultados para cada uno de los cuatro tópicos aplicables de Medición, análisis y mejora (pues el primer subítem corresponde a generalidades); posteriormente se presentan las conclusiones del artículo. Al final se encuentran las referencias bibliográficas de apoyo utilizadas y sugeridas al lector para profundizar en el tema.

\section{Propósito del estudio}

La expedición de la versión 2000 de las normas ISO 9000 cambió el referente del proceso al centrar su desarrollo en el establecimiento de Sistemas de gestión de la calidad y la evidencia, posterior a la certificación, de procesos de mejora continua.

Los procesos adelantados por las empresas a la luz de esta versión de la norma comenzaron a dar sus frutos hacia el año 2002, fecha en la cual se empezaron a expedir las certificaciones que reconocen el establecimiento del Sistema de gestión de la calidad en dichas empresas.

Muchas empresas de la ciudad de Ibagué han sido certificadas desde entonces, pero se desconocía la manera cómo se implementaron y desarrollaron las acciones encaminadas a asegurar y evidenciar la mejora continua del sistema.

Esta situación enmarcó el desarrollo del proyecto de investigación referenciado, el cual fue adelantado por el autor con la financiación del Comité Central de Investigaciones de la Universidad del Tolima (Uribe, 2011).

Con base en el proyecto mencionado, se propone presentar el análisis de los resultados obtenidos específicamente relacionados con el numeral 8 de la Norma ISO 9001:2000 Medición, análisis y mejora, sobre lo cual ésta establece en sus generalidades. En el Icontec (2001) se resalta lo siguiente:

“La organización debe planificar e implementar los procesos de seguimiento, medición, análisis y mejora necesarios para:

a) demostrar la conformidad del producto,

b) asegurarse de la conformidad del sistema de gestión de la calidad, y

c) mejorar continuamente la eficacia del sistema de gestión de la calidad.

Esto debe comprender la determinación de los métodos aplicables, incluyendo las técnicas estadísticas, y el alcance de su utilización." (p.117).

\section{Metodología}

El proyecto desarrollado corresponde a una investigación de tipo descriptiva pues se establecieron relaciones entre los diferentes rasgos inherentes al Sistema de gestión de la calidad de las empresas analizadas, lo cual supuso la descripción de los diferentes elementos y componentes del Sistema. El método de investigación es deductivo, debido a que a partir del referente general (la Norma ISO 9000) se analizó su aplicación en las empresas objeto de estudio.

La población estuvo conformada por las empresas de Ibagué que han obtenido la certificación ISO 9001:2000, con corte a junio de 2008; se utilizó la técnica de muestreo por conveniencia con base en la disposición de las empresas a participar en el suministro de la información. Se analizaron siete 
empresas de diferentes actividades, las cuales se detallarán más adelante.

Además de las fuentes secundarias consultadas (la familia de Normas ISO 9000, textos relacionados con el tema de la calidad, documentos escritos y páginas web de las empresas analizadas), se utilizó un cuestionario estructurado de 24 preguntas, diseñado con base en la Norma ISO 9004:2000. Directrices para la mejora del desempeño; el cual fue respondido por los gerentes o sus representantes ante el Sistema de calidad. Cada pregunta tiene una respuesta cualitativa y una valoración cuantitativa en una escala de 1 a 5 en la cual 5 representa la máxima calificación. Es importante aclarar que se trata de una autoevaluación por parte de las empresas, sin intervención del investigador para la calificación de dichos resultados.

Se presenta aquí el análisis de siete preguntas específicamente relacionadas con el ítem 8 de la norma, lo cual permite conocer de manera general la fundamentación de las mejoras realizadas por las empresas objeto de análisis en cuanto al seguimiento y medición, el control del producto no conforme, el análisis de datos, y la mejora.

\section{Resultados}

\section{Empresas estudiadas}

El estudio se desarrolló con base en la información suministrada por las siguientes siete empresas ibaguereñas (Uribe, 2011):

Aguialarmas S.A. se dedica a la prestación de servicios de vigilancia y seguridad privada y a la instalación y monitoreo de alarmas. Los procesos certificados fueron "Servicio de vigilancia y seguridad privada en las modalidades fija, móvil y escoltas, con armas y sin armas y con medios tecnológicos; Instalación y monitoreo de alarmas", cuya fecha de certificación fue el 23 de enero de 2008.

Cámara de Comercio de Ibagué, realiza las funciones públicas de registro mercantil y desarrolla actividades de capacitación y asesoría empresarial, certificación, y solución de conflictos. Los procesos que fueron certificados el 26 de mayo de 2004 fueron
"Registros públicos; Capacitación y asesoría empresarial; Certificación. Fue recertificada en mayo de 2007 y adicionados los procesos "Métodos alternos de solución de conflictos; Afiliación.

Centro de Productividad del Tolima se dedica a la identificación, formulación, ejecución, seguimiento y evaluación de proyectos de innovación, de investigación, de aplicación, y a la transferencia y difusión de tecnologías blandas. Certificó el proceso de "Identificación, formulación, ejecución, seguimiento y evaluación de proyectos de innovación, de aplicación, transferencia y difusión de tecnologías blandas." Fue certificada en noviembre de 2005 y recertificada (IS0 9001:2008, el 29 de mayo de 2009).

Colegio de La Presentación de Ibagué se dedica a la prestación del servicio de educación en los niveles preescolar, básica y media. Su proceso certificado fue "Diseño y prestación del servicio educativo en los niveles preescolar, básica y media", con fecha de certificación 26 de septiembre de 2007.

Confecciones Caribean S.A. se dedica a la producción de confecciones y prestación del servicio de maquila en confecciones. Fue certificada su proceso de "Producción y servicio de maquila de prendas de vestir en tejido plano", el 26 de febrero de 2003. Es la única de las empresas estudiadas que, estando en el período para hacerlo, no renovó su certificación.

Ingeniería y Construcciones Conseing Ltda. se dedica a la ejecución, construcción y mantenimiento de redes de telecomunicaciones y de obras civiles, así como consultoría y gestión de redes. El proceso certificado fue "Ejecución (construcción y mantenimiento) de redes de telecomunicaciones y obras civiles", en octubre de 2003; fue recertificada en octubre de 2006.

Trujillo Ortíz \& Cía. S. en C. presta los servicios de rectificación y reconstrucción de piezas y partes para automotores. Certificó el proceso "Servicio de rectificación de motores de combustión interna y de reconstrucción de componentes de maquinaria", cuya fecha fue 24 de septiembre de 2005; a la fecha del estudio se encontraba adelantando el proceso para la recertificación. 
Análisis parcial de los resultados, con base en cada aspecto indagado

La base para la obtención de la información con el objeto de realizar el análisis fue la Norma ISO 9004:2004, que "proporciona orientación sobre un rango más amplio de objetivos de un sistema de gestión de la calidad que la Norma ISO 9001, especialmente para la mejora continua del desempeño y de la eficiencia globales de la organización, así como de su eficacia." (ISO, 2008, p. 3) Ésta es una norma que se recomienda para las empresas que quieren impactar favorablemente sus procesos de mejora continua de su desempeño, esto es, ir más allá de la sola certificación obtenida con base en la ISO 9001.

En cuanto al numeral 8 de la Norma, a continuación se presentan las diferentes preguntas con los resultados obtenidos:

1. ¿Cómo se asegura la dirección de la recopilación de datos relacionados con el cliente para su análisis, con el fin de obtener información para mejoras? (Uribe, 2011, p.90)

Existen formatos que van desde la iniciación del servicio, en los cuales se registran todos los datos incluyendo sus consignas de seguridad (instrucciones específicas del cliente), como también mantenimiento del cliente con visitas periódicas programadas y formatos para registrar quejas y reclamos. Se recoge información por los medios ya mencionados, las auditorías internas establecen acciones de mejora; hay un procedimiento para mejora (no obligatorio) y existen formatos para su propuesta, análisis y aprobación. En otra empresa, por medios formales e informales que incluyen la retroalimentación verbal, telefónica y por escrito, así como la evaluación periódica de su satisfacción, y entre otros aspectos el cliente registra los puntos o áreas de mejora que el cliente considere. Se recoge la información, se hace análisis estadístico y análisis comparativo (hay 5 niveles o pasos: observar la tendencia, por qué -causas-, qué influencia tiene el resultado con relación al proceso y al resto de procesos, qué acciones se van a tomar de acuerdo al análisis, y seguimiento al resultado); entonces se produce mejora continua. Mediante formatos diligenciados: en este momento existen 123 formatos para registro vigentes. Por medio de encuestas con preguntas cerradas.

El $57 \%$ de las empresas se calificó con 5, el $29 \%$ se asignó 4 y el restante 14\% presentó una calificación de 3 , tal como se presenta en la figura 1.

Figura 1.

\section{Recopilación de datos relacionados con el cliente}

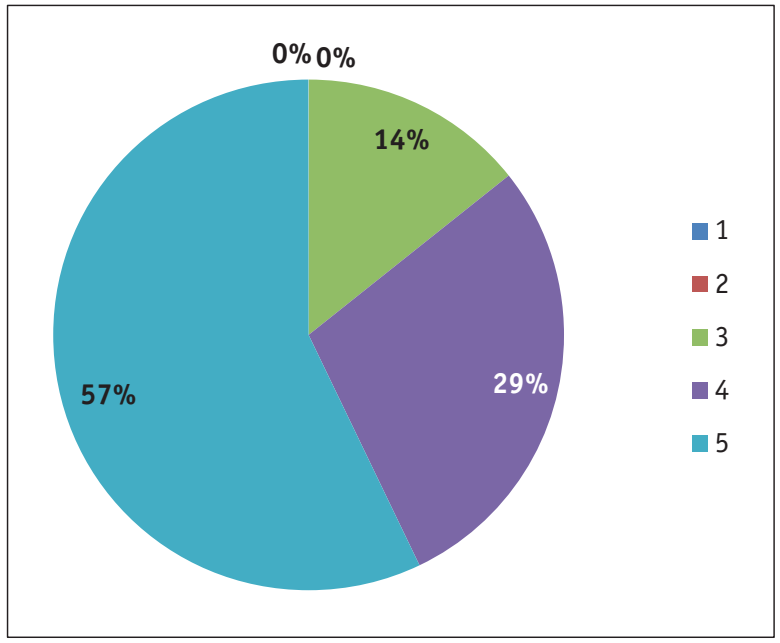

Fuente: Elaboración propia

2. ¿Cómo se obtienen los datos de otras partes interesadas para análisis y posibles mejoras? (Uribe, 2011, p. 91)

Mediante comunicaciones; no hay encuestas pues éstas solo son para el cliente. A nivel de Comfecámaras existen comités como los administrativos, jurídicos, desarrollo empresarial y legal, en los cuales se manejan todos los temas inherentes; de allí se toman las mejores prácticas (retroalimentación). En otra organización se hace de manera formal e informal, así mismo, con respecto a otras partes interesadas como la Junta directiva se obtiene retroalimentación constante en las reuniones de Consejo y Asamblea. Mediante las entradas para el diseño del currículo (voz del usuario, evaluación institucional de padres de familia, estudiantes y profesores); lineamientos institucionales, y buzón de sugerencias. En otras mediante actas de comités y realimentación pe- 
riódica con el cliente. Por medio de un sistema de sugerencia tanto del personal interno como externo.

La figura 2 muestra que el $57 \%$ de los encuestados se calificó con 5, el $29 \%$ con 3 y el restante $14 \%$ se asignó 4.

\section{Figura 2.}

Obtención de datos de otras partes interesadas

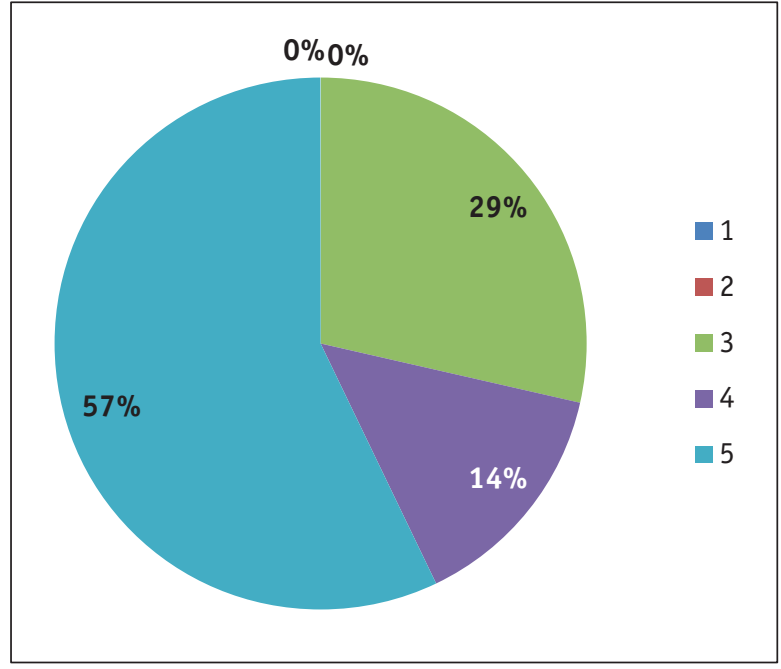

Fuente: Elaboración propia

3. ¿Cómo usa la organización las metodologías de autoevaluación del sistema de gestión de la calidad para la mejora de la eficacia y la eficiencia globales de la organización? (Uribe, 2011, p.92)

No se utilizan metodologías; solo las auditorías internas y las revisiones gerenciales. La información se recopila y se evalúa frente al cumplimiento de la normatividad legal existente; revisión de la Contraloría General de la República y la Superintendencia de Industria y Comercio para las funciones delegadas por el Estado. Mediante las auditorías internas se realiza una evaluación sistemática del Sistema de gestión de la calidad y es en este ejercicio en el que se descubren nuevos aspectos a mejorar; otra herramienta posterior a ésta es la Revisión por la Dirección. De la autoevaluación de cada proceso surge la acción de mejoramiento, los procesos tienen procedimientos; la sumatoria de la evaluación del proceso va en la de los otros, además de la revisión por la dirección. En una de las empresas no se ha implementado metodología de autoevaluación pues se espera que el sistema de gestión sea más maduro. Se usan auditorías internas de calidad, con el fin de evaluar el funcionamiento de los procesos y el Sistema de Gestión de la Calidad en general y sus actividades correspondientes; de esta manera se irán identificando los puntos favorables y críticos y se llevaría a realizar las respectivas modificaciones.

Figura 3.

\section{Uso de metodologías de autoevaluación del sistema}

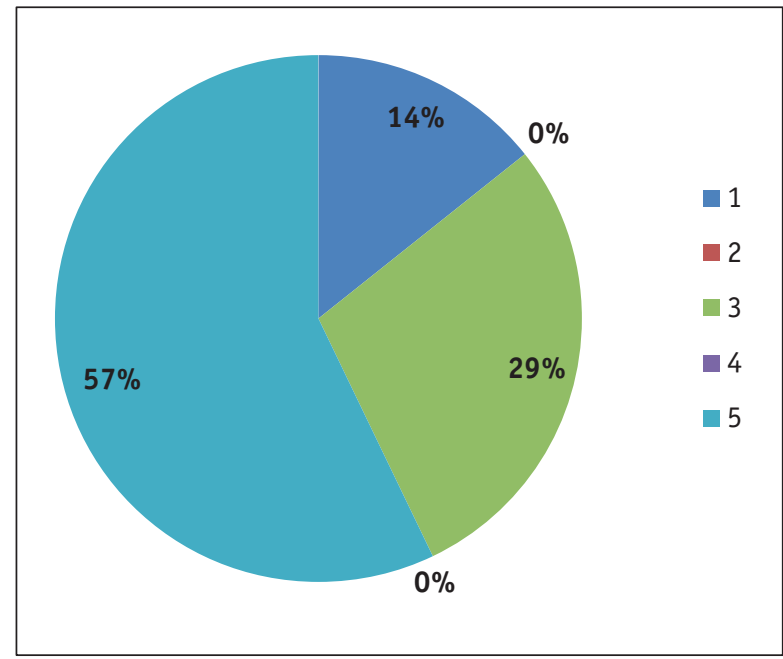

Fuente: Elaboración propia

El 57\% de los encuestados se asignó una calificación de 5, el 29\% de ellos se calificó 3 y un 14\% presentó una calificación de 1 . Su representación se observa en la figura 3.

4. ¿Cómo analiza la organización las no conformidades para aprendizaje y mejora del proceso y del producto? (Uribe, 2011, p.93)

Se utiliza un análisis de causas (los 3 por qué), para mejora se utilizan las herramientas para soporte al cliente. Se analiza mediante quejas y reclamos; y la determinación y análisis de producto no conforme: con base en ello se toman las acciones preventivas, 
correctivas y de mejora. Las no conformidades se revisan con el responsable del proceso y las personas que considere apropiadas y que estén relacionadas con el hallazgo, y si es posible, con la colaboración de un auditor, luego se envían al auditor que las generó para que evalúe si realmente atacan la causa y les dé vía libre para ejecutar las acciones.

En otra organización se trabaja la espina de pescado pues es más fácil (se intentó los tres por qué). En otro caso se analiza mediante un instructivo propio sobre análisis de causas denominado los siete reportes. De acuerdo con los resultados de las auditorías internas, se realizan mejoras a las no conformidades existentes, en un período determinado.

El $57 \%$ de los encuestados se calificó 5 , en tanto que el restante $43 \%$ lo hizo con 4 . En la figura 4 se aprecia este resultado.

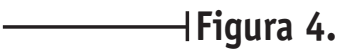

\section{Análisis de las no conformidades}

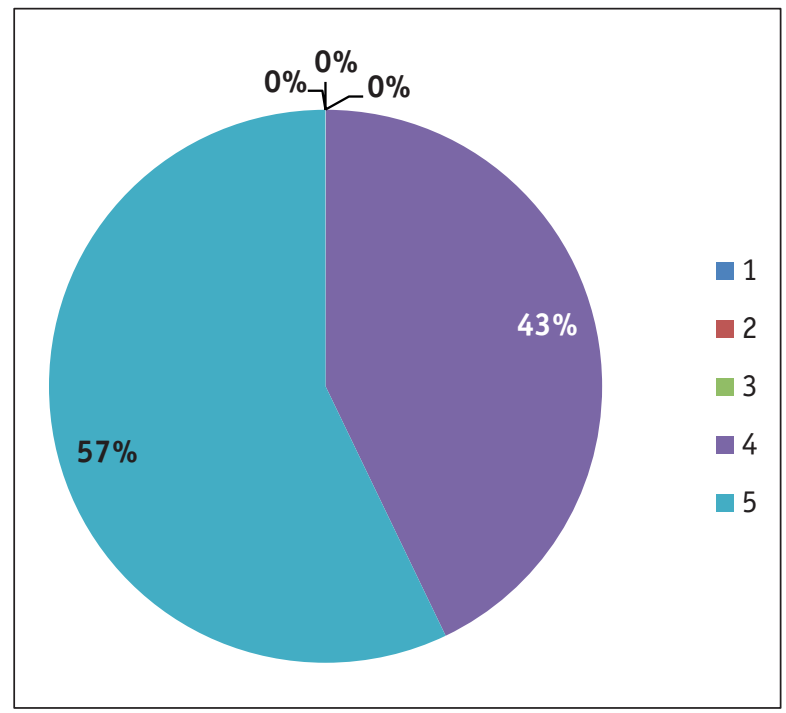

Fuente: Elaboración propia

5. ¿Cómo usa la dirección las acciones correctivas para evaluar y eliminar los problemas registrados que afectan su desempeño? (Uribe, 2011, p.95)

Se recibe la no conformidad, se le realiza su análisis y su tratamiento inmediato y si requiere plan de acción se formula. Es perentorio corregir (por el cumplimiento de la normatividad legal) pues puede implicar sanciones de tipo legal; se plantean, analizan, evalúan e implementan; si se requiere asignación de dinero se hace.

El Coordinador de calidad sigue de cerca el proceso para evaluar su efectividad y asegurarse de que se elimine completamente el problema. Se acude a las 5M; ayuda a determinar qué parte falló; de allí se focaliza el trabajo en la causa real y se expresa que no se vuelva a repetir. Se hace el seguimiento de las actividades encaminadas a satisfacer las no conformidades, desde la reflexión y no desde el método. Se usa por medio de una metodología basada en un diagrama de flujo de las causas y efectos $y / 0$ cuando sea aplicable lluvia de ideas para la toma de decisiones, se incluye la revisión periódica de las acciones con el fin de determinar su continua eficacia.

En esta pregunta el $72 \%$ de las empresas encuestadas se asignó la máxima calificación de la escala: 5 , un $14 \%$ se calificó con 4 e igual porcentaje lo hizo con 3. Se observa a continuación en la figura 5.

Figura 5.

\section{Uso de las acciones correctivas para evaluar y eliminar problemas}

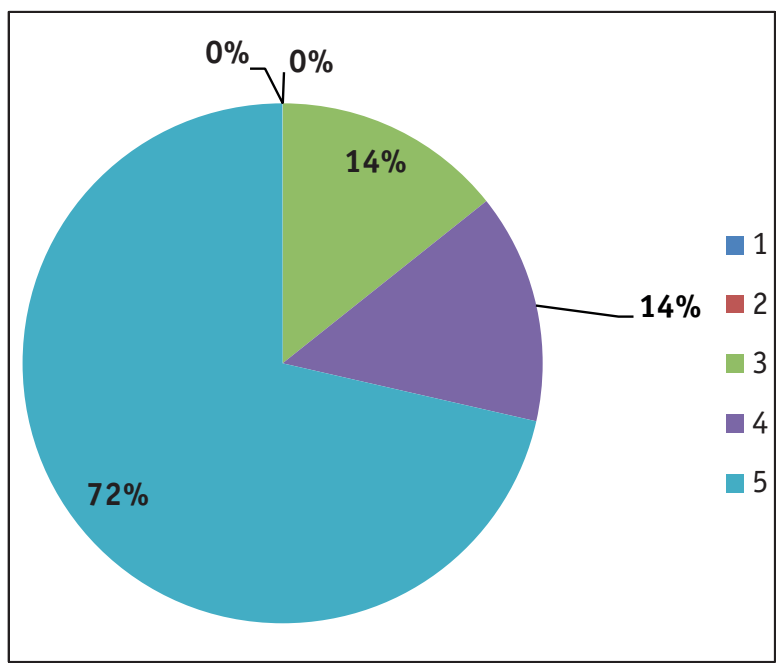

Fuente: Elaboración propia 
6. ¿Cómo usa la dirección las acciones preventivas para la prevención de pérdidas? (Uribe, 2011, p.96)

\section{- Figura 6.}

\section{Uso de las acciones preventivas para preven- ción de pérdidas}

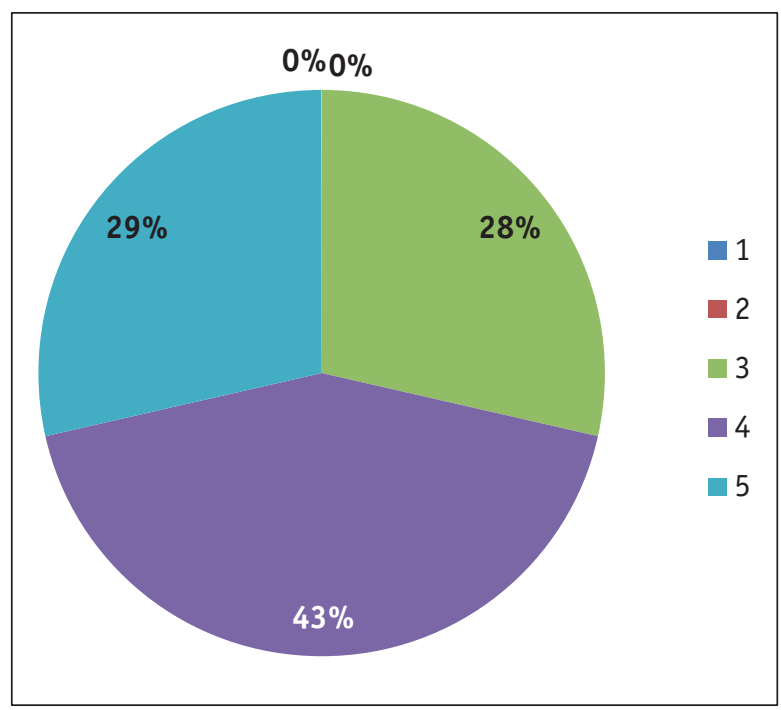

Fuente: Elaboración propia

Los dueños de procesos formulan actividades para acciones preventivas; igualmente se pueden recibir de los clientes. Todo se analiza en las reuniones administrativas programadas. En una de las organizaciones estudiadas existe debilidad en la identificación de acciones preventivas; se debe fortalecer este aspecto Identificación - Implementación. Se generan acciones preventivas encaminadas a la eliminación de causas de problemas potenciales y se revisan o verifican que los resultados sean los esperados. Se le hace 5M (no siempre); se focaliza la energía en la causa potencial (se previene, antes de...); se detectan anticipadamente riesgos; la acción preventiva la elabora el dueño del proceso. Dependiendo de la naturaleza del proyecto se trabaja en la minimización de la pérdida o en la anulación de la misma; ello depende de los agentes externos como las condiciones de mercado, balanza, etc. En otro caso, se hace por medio de un diagrama de flujo para la descripción del procedimiento de acciones preventivas, en el cual se identifican cada una de las actividades, los responsables y los registros utilizados en este procedimiento.

El $43 \%$ de las empresas encuestadas se calificó con 4 , el $29 \%$ con 5 y el restante $28 \%$ lo hizo con 3 . Estos resultados se observan en la figura 6 .

7. ¿Cómo se asegura la dirección el uso sistemático de métodos y herramientas para mejorar el desempeño de la organización? (Uribe, 2011, p.97)

Poco se utilizan; la metodología es interna; sin embargo la organización se está preparando para aplicarlas (se utiliza la metodologías de los 3 por qué). Con seguimiento permanente por parte de la alta dirección, a todos los procesos, funciones, y cumplimiento de normatividad. En el caso de otra organización, se asegura mediante la definición del espacio "lunes de calidad", la revisión por la dirección, las auditorías internas, la capacitación constante del personal en nuevas metodologías y herramientas y mediante la provocación constante de un espíritu de mejora. La reiteración de las charlas (para optimizar el método pedagógico) desde diferentes miradas; generalmente se utiliza el análisis de Pareto y la Espina de pescado. Mediante sus sistema de comunicación interna F-87, Distribución certificación de documentos y formatos. Se asegura por medio de resultados arrojados por las diferentes encuestas y realizadas tanto al personal interno como externo y al seguimiento periódico de los resultados.

Para esta pregunta el $43 \%$ de las empresas se calificaron con 5, igual porcentaje se asignaron una calificación de 4, y el 14\% lo hizo con 3 . A continuación se observa de manera gráfica en la figura 7.

\section{Análisis de los resultados, de acuerdo con}

los ítems del numeral 8. Medición, análisis y mejora

Para finalizar, es importante relacionar los aspectos analizados anteriormente con el subítem de la norma correspondiente: 
Figura 6.

\section{Uso sistemático de métodos y herramientas para mejorar el desempeño}

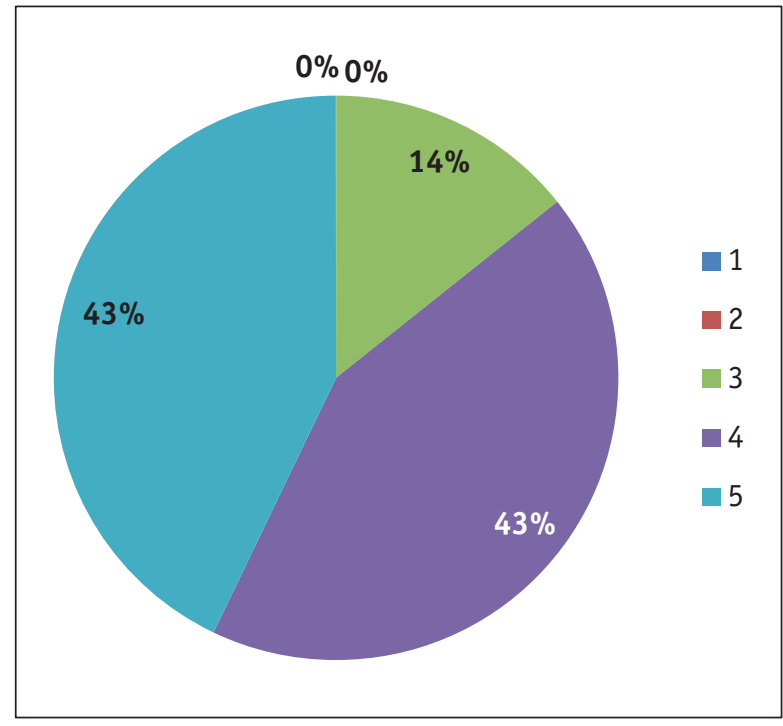

Fuente: Elaboración propia

Ítem de la Norma 8.2 Seguimiento y medición

En consideración a lo establecido en la Norma (Icontec, 2001), se refiere a la medición de la satisfacción del cliente, la realización de auditorías internas, el seguimiento y medición de los procesos, y el seguimiento y medición de los productos.

De acuerdo con las respuestas obtenidas de las preguntas 1,2 y 3 , el cumplimiento de este tópico se realiza mediante la utilización de instrumentos formales e informales como formatos y retroalimentación verbal, respectivamente. Se realiza análisis estadístico, una vez recogida la información, y se toman decisiones de mejoramiento. Estas actividades se realizan con los clientes y con las demás partes interesadas en la organización; para este último caso se utilizan también reuniones de comités y juntas directivas, así como buzones de sugerencias. Las metodologías utilizadas son las auditorías internas de calidad y la revisión por la dirección, además de la autoevaluación de los procesos.
Ítem de la Norma 8.3 Control del producto no conforme

En este caso es necesario que la organización identifique y controle el producto que no cumple con las especificaciones del cliente $y / 0$ legales, mediante un procedimiento que debe estar evidenciado en el Sistema.

En términos generales las empresas estudiadas identifican las no conformidades escuchando la voz del cliente y utilizando diferentes herramientas, sobre cuyos resultados se realizan análisis con las personas que intervienen en los procesos pertinentes y se toman decisiones y emprenden acciones orientadas a la mejora. Este tópico se encuentra reflejado en la pregunta 4.

\section{Ítem de la Norma 8.4 Análisis de datos}

En este tópico (IS0, 2000) la organización debe recopilar y analizar los datos para evidenciar la idoneidad y eficacia del Sistema y para evaluar en dónde puede realizar su mejora continua.

En las respuestas a la pregunta 7 se observa la utilización de diversas técnicas y herramientas para el adecuado análisis y tratamiento de los datos pertinentes para la mejora de la eficacia del Sistema: se generan espacios para que el talento humano de la organización realice los análisis y sugerencias al respecto, mediante seguimiento y revisión por parte de la dirección, la aplicación de métodos pedagógicos, y seguimiento periódico a los resultados. Para ello, se utilizan herramientas como los tres por qué, el análisis de Pareto, y la espina de pescado.

\section{Ítem de la Norma 8.5 Mejora}

Este tópico comprende los aspectos directamente relacionados con la mejora continua, las acciones correctivas, y las acciones preventivas; las cuales deben implementarse en la organización para mejorar la eficacia del Sistema.

Con base en las respuestas a las preguntas 5 y 6 se puede establecer que las organizaciones reciben la 
no conformidad, realizan de manera inmediata su análisis y emprenden la acción correctiva, cuando es necesario se elabora un plan de acción. Para el análisis se utilizan herramientas como las $5 \mathrm{M}$, el diagrama de flujo y la lluvia de ideas. Estas herramientas son utilizadas también para las acciones preventivas, pero en este caso el responsable es el dueño del proceso quien inicia el proceso de oficio o por recomendación de los clientes, para eliminar los problemas potenciales, a partir de sus causas.

\section{Conclusión}

En general, existe una alta valoración en la manera como las empresas califican su gestión frente al Sistema, ésta fluctúa en términos generales entre 4 y 5, máximos valores de la escala propuesta para la autoevaluación de las organizaciones.

La alta dirección se asegura que los elementos de entrada de los procesos de realización tienen en cuenta las necesidades de los clientes y de otras partes interesadas (Uribe, 2011) mediante los procedimientos establecidos en los cuales se detalla el paso a paso desde la captación de los requerimientos del cliente hasta la prestación del servicio. Se utilizan planes de calidad, cuando es pertinente, y se llevan matrices de documentos.

La dirección se asegura de la recopilación de datos relacionados con el cliente con el fin de obtener información para mejoras (Uribe, 2011), utilizando formatos desde la iniciación del servicio, como también mantenimiento del cliente con visitas periódicas programadas y formatos para registrar quejas y reclamos. De igual manera mediante instrumentos no formales como la retroalimentación verbal y telefónica.

Los datos de otras partes interesadas para análisis y posibles mejoras se obtienen mediante comunicaciones, comités, juntas directivas, y buzones de sugerencias para personal interno y externo.

No se evidencian metodologías de autoevaluación del sistema de gestión de la calidad, diseñadas para la mejora de la eficacia y la eficiencia globales de la organización (Uribe, 2011), pero se utilizan las auditorías de calidad, las revisiones por la gerencia, y la autoevaluación de los procesos
La organización analiza las causas de las no conformidades para aprendizaje y mejora del proceso y del producto mediante un análisis de causas (los 3 por qué), quejas y reclamos, auditorías internas, espina de pescado, instructivo específico denominado los 7 reportes, entre otros.

La dirección usa las acciones correctivas para evaluar y eliminar los problemas registrados que afectan su desempeño (Uribe, 2011), a partir de la recepción de las no conformidades, el diseño de planes de acción, el uso de la herramienta 5M o la lluvia de ideas; se realiza seguimiento de las actividades encaminadas a satisfacer las no conformidades, desde la reflexión y no desde el método.

La dirección usa las acciones preventivas para la prevención de pérdidas mediante los dueños de los procesos quienes formulan actividades para acciones preventivas. Se analizan en reuniones administrativas; algunas veces se utiliza el análisis de 5M, otras un diagrama de flujo para la descripción del procedimiento de acciones preventivas. No obstante, es manifiesto para algunos que existen debilidades en este aspecto.

La dirección se asegura del uso sistemático de métodos y herramientas para mejorar el desempeño de la organización (Uribe, 2011) con seguimiento permanente a todos los procesos, funciones y cumplimiento de la normatividad; también mediante el desarrollo del espacio lunes de calidad, la revisión por la dirección, las auditorías de calidad, el uso del análisis de Pareto y de la Espina de pescado. Vale decir que en algunos casos no se utilizan métodos sistemáticos para este fin.

\section{Referencias Bibliográficas}

ICONTEC. (2001). Guía para las pequeñas empresas sobre la Norma ISO 9001:2000. Bogotá: Autor.

ISO (2000). Norma técnica colombiana NTC-ISO 9004, Sistemas de gestión de la calidad. Directrices para la mejora del desempeño. Bogotá: ICONTEC.

ISO (2008). Norma técnica colombiana NTC-ISO 9001, Sistemas de gestión de la calidad. Requisitos. Bogotá: ICONTEC.

Uribe, M. E. (2011). Los Sistemas de Gestión de la Calidad: -el enfoque teórico y la aplicación empresarial-. Ibagué: Universidad del Tolima. p. 72-98. 